\title{
Sector Financiero Popular y Solidario: cooperativas de ahorro y crédito del segmento 5 en Ecuador
}

\section{Popular and Solidary Financial Sector: Savings and Credit Cooperatives of Segment 5 in Ecuador}

Ángel Eduardo Rodríguez Solarte. ${ }^{1}$, Jenny Isabel Chávez Rojas. ${ }^{2}$, Víctor Gabriel Ávalos Peñafiel. ${ }^{3}$, Roberto Fabián Sánchez Chávez. ${ }^{4}$

Recibido: 20-04-2019 / Revisado: 24-05-2019 /Aceptado: 25-06-2019/ Publicado: 30-07-2019

\begin{abstract}
DOI: https://doi.org/10.33262/cienciadigital.v3i3.3.809

In 2015, by resolution of the Monetary and Financial Politics issues, "The Standard for the Segmentation of Entities of the Popular and Solidary Financial Sector", which establishes in its article 1, that the segmentation of the Cooperatives of Savings and Credit, will be made according to the type and balance of its assets. The present academic work is directed to segment 5 , where they will evaluate their most representative accounts in order to establish the suitability or not of their existence within the market, as well as the level of confidence generated by these organizations within the financial system of the country, Taking into account their financial situation and statistics of liquidation the processes that have occurred in this segment.
\end{abstract}

Keywords: Financial System, popular finance, cooperatives of saving and credit, liquidations.

\section{Resumen}

En el año 2015, por resolución de la Junta de Política Monetaria y Financiera se expide "La Norma para la Segmentación de las Entidades del Sector Financiero Popular y Solidario" donde se establece en su artículo 1, que la segmentación de las Cooperativas de Ahorro y Crédito, se realizará de acuerdo al tipo y al saldo de sus activos. El presente trabajo está dirigido al segmento 5, donde se evaluarán sus cuentas más representativas en pro de establecer la conveniencia o no, de su existencia dentro del mercado, así como el nivel de confianza que generan estas organizaciones dentro del sistema financiero del país, teniendo

\footnotetext{
${ }^{1}$ Ingeniero de Empresas. Magister en Pequeñas y Medianas Empresas Mención Finanzas, ESPOCH, Finanzas, Administración de empresas, Riobamba, edu29ro@ hotmail.com

${ }^{2}$ Economista. Master en Dirección de Empresa Mención Proyectos, ESPOCH, Finanzas, Administración de empresas, Riobamba, jennisa05@yahoo.es

${ }^{3}$ Licenciado en Contabilidad y Auditoría. Magister en Pequeñas y Medianas Empresas Mención Finanzas, Gaboavalos1972@hotmail.com

${ }^{4}$ Abogado de los Juzgados y República del Ecuador. Magister en Derecho Procesal Civil, ESPOCH, Finanzas, Administración de empresas, Riobamba, Robertonga@ hotmail.es
} 
en cuenta su salud financiera y estadísticas de procesos de liquidación que se hayan dado en este segmento.

Palabras Claves: Sistema Financiero, finanzas populares, cooperativas de ahorro y crédito, liquidaciones.

\section{Introducción}

La economía popular y solidaria, en adelante (EPS) es la forma de organización económica, donde sus integrantes, individual o colectivamente, organizan y desarrollan procesos de producción, intercambio, comercialización, financiamiento y consumo de bienes y servicios, para satisfacer necesidades y generar ingresos; para conceptualizar y aplicar normas a esta economía es importante identificar las organizaciones que pertenecen al sector financiero y las que no. El Sector Financiero Popular y Solidario, en adelante (SFPS), se rige al Código Monetario, teniendo en cuenta que la Junta de Política Monetaria será quien se atribuya expedir las diferentes normas para las instituciones que conforman el sistema financiero; para el año 2015 se expide la "Norma para la Segmentación del Sector Financiero Popular y Solidario", siendo así que la segmentación consta de 5 grupos, partiendo del artículo 447 del Código Orgánico Monetario y Financiero, donde el segmento 1 corresponde a entidades con un nivel de activos superior a USD 80'000.000,00; posteriormente la cantidad aumentaría según determine la ley y condiciones del país, teniendo en cuenta las variaciones del índice del precio al consumidor, es así que inicia el sector cooperativista con un enfoque diferente en relación al tipo y cantidad de activos. A continuación, se detalla la segmentación, que se tendrá en cuenta durante el análisis específico del segmento 5.

Tabla 1.- Segmentación de Cooperativas de Ahorro y Crédito

\begin{tabular}{cc}
\hline Segmento & Activos (USD) \\
\hline 1 & Mayor a 80000000 \\
2 & Mayor a 20000000 hasta 80000000 \\
3 & Mayor a 5000000 hasta 20000000 \\
4 & Mayor a 1000000 hasta 5000000 \\
5 & Hasta 1000000 \\
\hline
\end{tabular}

Fuente: Junta de Política Monetaria - Autores

En el país existe un gran número de Cooperativas de Ahorro y Crédito, siendo en total 696 entidades [1], y que gracias a la segmentación son fáciles de ubicar y definir el impacto que tienen dentro del sistema financiero; antes de la aplicación de la ley se contaban alrededor de 1164 entidades, teniendo que limitar y liquidar sus funciones a muchas de ellas por no cumplir con los requerimientos establecidos.

Tabla 2.- Número de Entidades

\begin{tabular}{ccc}
\hline Segmento & No de Entidades & \% de Representación \\
\hline 1 & 26 & $3,74 \%$ \\
2 & 33 & $4,74 \%$ \\
3 & 84 & $12,07 \%$ \\
4 & 183 & $26,29 \%$ \\
5 & 370 & $53,16 \%$ \\
Total & 696 & $100 \%$ \\
\hline
\end{tabular}

Fuente: Junta de Política Monetaria - Autor 
En la relación de representación porcentual tenemos que el segmento 5 corresponde a un 53,16\% en número de entidades de un total de 696, siendo por tanto la mayor representación con 370 Cooperativas de Ahorro y Crédito, sin embargo, con una cantidad y tipo de activo igual o menor a USD 1000000 (un millón de dólares). Con esta relación se ha identificado a estas organizaciones pequeñas pero numerosas, las mismas que sin un control adecuado (de sus Estados Financieros, balances, apego a la ley) y reglas claras pueden generar desconfianza en el sistema, creando un riesgo reputacional para los otros segmentos.

Teniendo en cuenta la cantidad de sus activos, es meritorio analizar si estas cuentas representan y avalan la existencia de estas entidades; si se cuenta con una relación mínima de 1:1 con los pasivos y si sus utilidades demuestran una correcta administración de los recursos. La siguiente tabla representa el total de Activos, Pasivos, Patrimonio y Capital Social generados en el año 2016 por las COAC's en sus diferentes segmentos.

Tabla 3.- Representación porcentual en cantidad de Activos (Cifras relacionadas a millones de dólares)

\begin{tabular}{crrrrrr}
\hline Cuentas & Segmento 1 & Segmento 2 & Segmento 3 & Segmento 4 & Segmento 5 & \multicolumn{1}{c}{ Total } \\
\hline ACTIVOS & $6.641,61$ & $1.372,06$ & 946,91 & 427,09 & 127,16 & $9.514,82$ \\
$\%$ & 69,80 & 14,42 & 9,95 & 4,49 & 1,34 & 100 \\
PASIVOS & $5.664,52$ & $1.148,97$ & 775,62 & 343,62 & 95,04 & $8.027,76$ \\
$\%$ & 70,56 & 14,31 & 9,66 & 4,28 & 1,18 & 100 \\
PATRIMONIO & 977,09 & 223,09 & 171,29 & 83,47 & 32,12 & $1.487,06$ \\
$\%$ & 65,71 & 15,00 & 11,52 & 5,61 & 2,16 & 100 \\
CAPITAL & 427,05 & 105,44 & 87,70 & 52,86 & 19,40 & 692,45 \\
SOCIAL & 61,67 & 15,23 & 12,67 & 7,63 & 2,80 & 100 \\
$\%$ & &
\end{tabular}

Fuente: SEPS (Boletín abril 2017/ Al 31 de diciembre. 2016) - Autor

El segmento 5 representa el 1,34\% del valor total en Activos generados en el periodo, el 1,18\% del valor de Pasivos, el 2,16\% del Patrimonio y un 2,80\% del Capital Social generado en el año 2016; teniendo en cuenta que su constitución o valor en activos representa en un millón de dólares o menos de este valor, los porcentajes no son alarmantes, sin embargo, es el número de entidades lo que no representa un buen síntoma financiero. Sin embargo, es importante detallar las cuentas que involucren la liquidez, la solvencia, la morosidad y la utilidad que tienen estas organizaciones, cumpliendo los objetivos para los cuales fueron creadas y sobre todo si garantizan su permanencia en el mercado.

\section{Metodología}

La metodología empleada en el trabajo hace referencia a una investigación acción con un método analítico sintético con un enfoque cuali - cuantitavo. Para lo cual trabajó con indicadores financieros como herramientas construidas utilizando, la información financiera de las entidades, y son necesarias para medir la estabilidad, la capacidad de endeudamiento, la capacidad de liquidez, los rendimientos y las utilidades de la entidad, a través de la interpretación de las cifras, de los resultados y de la información en general [2]. Para proceder con este estudio se identificó los datos reales de 
un periodo transcurrido en este caso es el periodo enero - diciembre de 2016, donde tenemos un resumen del balance anual de los segmentos. Se identificó el número de entidades en procesos de liquidación con el catastro que muestra la Superintendencia de Economía popular y Solidaria y su localización se realizó mediante la geo - referenciación de la misma institución.

\section{1.- Indicadores Financieros}

Teniendo en cuenta este Balance resumen, podemos desglosar las cuentas representativas para el análisis financiero de la situación del segmento 5 dentro del SFPS

Tabla 4.- Balance periodo 2016

\begin{tabular}{lcccccc}
\hline & Segmento 1 & Segmento 2 & Segmento 3 & Segmento 4 & Segmento 5 & Total \\
\hline ACTIVOS & $6.641,61$ & $1.372,06$ & 946,91 & 427,09 & 127,16 & $9.514,82$ \\
PASIVOS & $5.664,52$ & $1.148,97$ & 775,62 & 343,62 & 95,04 & $8.027,76$ \\
PATRIMONIO & 977,09 & 223,09 & 171,29 & 83,47 & 32,12 & $1.487,06$ \\
CAPITAL SOCIAL & 427,05 & 105,44 & 87,70 & 52,86 & 19,40 & 692,45 \\
CRÉDITOS (CUENTA 14) & $4.108,77$ & 991,13 & 681,29 & 305,87 & 78,37 & $6.165,43$ \\
DEPÓSITOS (CUENTA 21) & $5.307,12$ & $1.054,21$ & 678,72 & 288,99 & 81,47 & $7.410,51$ \\
\hline
\end{tabular}

Fuente: SEPS (Boletín abril 2017/ Al 31 de diciembre. 2016) - Autor

Cartera colocada.

Uno de los objetivos principales de toda entidad financiera es captar y colocar recursos, para que la entidad pueda mantenerse dentro del mercado; al hablar del SFPS a más de este objetivo lo que se busca es el desarrollo social, la integración de los más pequeños actuando con principios de solidaridad, brindando a sus socios créditos en diferentes segmentos, para el año 2016 se contó con una cartera de crédito de USD 6.165.431.523,13 donde el segmento 5 representa el 1,30\% del total de esta cartera colocada.

Tabla 5.- Cartera de Créditos de 2016 de las Cooperativas de Ahorro y crédito (5 Segmentos)

\begin{tabular}{lcccccc}
\hline & Segmento 1 & Segmento 2 & Segmento 3 & Segmento 4 & Segmento 5 & Total \\
\cline { 2 - 6 } $\begin{array}{c}\text { CARTERA DE } \\
\begin{array}{c}\text { CRÉDITOS } \\
2016\end{array}\end{array}$ & $4.108 .769 .400,61$ & $991.129 .064,96$ & $681.290 .211,24$ & $305.873 .052,67$ & $78.369 .793,65$ & $6.165 .431 .523,13$ \\
& & & & & \\
& $66,64 \%$ & $16,08 \%$ & $11,05 \%$ & $4,96 \%$ & $1,27 \%$ & 100,00 \\
\hline
\end{tabular}

Fuente: SEPS (Boletín abril 2017/ al 31 de diciembre 2016) - Autor

Teniendo una segregación también en los tipos de crédito por cada segmento de: 
Tabla 6.- Tipos de Crédito por Segmento

\begin{tabular}{lcccccc}
\hline Tipos de Crédito & Seg 1 & Seg 2 & Seg 3 & Seg 4 & Seg5 & Promedio \\
\hline Crédito Comercial Prioritario & 112,71 & 23,96 & 4,38 & 1,74 & 1,23 & 144,01 \\
Crédito De Consumo Prioritario & $2.289,47$ & 473,04 & 292,70 & 112,69 & 23,86 & $3.191,76$ \\
Crédito Inmobiliario & 283,84 & 53,24 & 20,24 & 7,27 & 2,05 & 366,64 \\
Microcrédito & $1.597,86$ & 504,67 & 381,44 & 194,32 & 50,92 & $2.729,22$ \\
Crédito Productivo & 2,32 & 0,00 & 0,07 & 0,05 & 0,18 & 2,62 \\
Crédito Comercial Ordinario & 0,62 & 0,00 & 0,00 & 0,05 & 0,35 & 1,02 \\
Crédito De Consumo Ordinario & 122,02 & 15,73 & 18,77 & 4,74 & 4,21 & 165,47 \\
Crédito De Vivienda De Interés Público & 0,00 & 0,00 & 0,14 & 0,13 & 0,02 & 0,30 \\
Crédito Educativo & 0,00 & 0,01 & 0,07 & 0,22 & 0,00 & 0,30 \\
\hline
\end{tabular}

Fuente: SEPS (Boletín abril 2017/ al 31 de diciembre 2016) - Autor

La siguiente tabla representa los índices de morosidad obtenidos en los tipos de créditos, (tabla 5), otorgados en los cinco segmentos:

Morosidad de la cartera.

Tabla 7.- Índices de Morosidad

\begin{tabular}{lcccccc}
\hline Créditos & Seg 1 & Seg 2 & Seg 3 & Seg 4 & Seg5 & Promedio \\
\hline Crédito Comercial Prioritario & $6,97 \%$ & $28,31 \%$ & $15,74 \%$ & $37,05 \%$ & $24,79 \%$ & $11,30 \%$ \\
Crédito De Consumo Prioritario & $4,14 \%$ & $7,34 \%$ & $8,28 \%$ & $10,77 \%$ & $12,98 \%$ & $5,29 \%$ \\
Crédito Inmobiliario & $2,87 \%$ & $7,49 \%$ & $6,19 \%$ & $7,93 \%$ & $29,12 \%$ & $3,97 \%$ \\
Microcrédito & $7,92 \%$ & $12,73 \%$ & $13,98 \%$ & $17,21 \%$ & $18,10 \%$ & $10,51 \%$ \\
Crédito Productivo & $0,00 \%$ & $0,00 \%$ & $0,00 \%$ & $3,42 \%$ & $5,18 \%$ & $0,43 \%$ \\
Crédito Comercial Ordinario & $0,00 \%$ & $0,00 \%$ & $0,00 \%$ & $0,00 \%$ & $10,20 \%$ & $5,84 \%$ \\
Crédito De Consumo Ordinario & $0,51 \%$ & $0,55 \%$ & $3,26 \%$ & $3,14 \%$ & $3,05 \%$ & $0,97 \%$ \\
Crédito De Vivienda De Interés Público & $0,00 \%$ & $0,00 \%$ & $0,07 \%$ & $0,01 \%$ & $0,00 \%$ & $0,01 \%$ \\
Crédito Educativo & $0,00 \%$ & $55,73 \%$ & $16,48 \%$ & $84,18 \%$ & $0,00 \%$ & $66,37 \%$ \\
\hline
\end{tabular}

Fuente: SEPS (Boletín abril 2017/ al 31 de diciembre 2016) - Autor

Para el cálculo total de la morosidad de la cartera utilizamos la cartera total donde las subdivisiones serán: la Cartera que no devenga intereses más la cartera vencida dividiendo este resultado para la cartera neta; lo cual nos indica que mientras menor sea el valor de este índice mejor será la situación de la institución en el sistema.

$$
\text { Morosidad Cartera }=\frac{(\text { Cartera que no devenga intereses }+ \text { cartera vencida })}{\text { Cartera Neta }}
$$

Tabla 8.- Morosidad por Segmentos

\begin{tabular}{cccccc}
\hline Segmento 1 & Segmento 2 & Segmento 3 & Segmento 4 & Segmento 5 & Promedio \\
\hline $5,40 \%$ & $10,26 \%$ & $11,16 \%$ & $14,67 \%$ & $16,16 \%$ & $7,40 \%$
\end{tabular}

Fuente: SEPS (Boletín abril 2017/ al 31 de diciembre 2016) - Autor

Se identifica al segmento 5 con el mayor valor de morosidad en el Sistema Financiero Popular y Solidario, con un 16,16\% superando incluso el promedio de este indicador que es el 7,40\%. 
La liquidez.

En instituciones del sistema financiero, se toman en cuenta los fondos disponibles con los que cuenta la institución, dividiendo para el total de depósitos a corto plazo, donde un mayor valor de esta relación, refleja una mejor posición de liquidez en el sistema

$$
\text { Liquidez }=\frac{\text { Fondos Disponibles }}{\text { Total Depósitos a Corto Plazo }}
$$

Dando como resultado, que el segmento 5 cuenta con un $50,6 \%$ de liquidez contrastando el promedio para los demás segmentos que alcanzan un $28,8 \%$

La solvencia.

Para este indicador tomamos en cuenta la Cartera Improductiva sobre el Patrimonio de la entidad; mientras el valor de esta relación sea mayor, reflejará una mejor posición de la entidad en relación al sistema.

$$
\text { Solvencia }=\frac{\text { Cartera Improductiva }}{\text { Patrimonio }}
$$

Dando como resultado, que el segmento 5 cuenta con un $41,7 \%$ de solvencia contrastando el promedio para los demás segmentos que alcanzan un $32,9 \%$.

\begin{tabular}{|c|c|c|c|c|c|}
\hline Indicadores financieros 2015 & $\begin{array}{c}\text { Total } \\
\text { Segmento } \\
5\end{array}$ & $\begin{array}{l}\text { Total } \\
\text { SFPS }\end{array}$ & Indicadores financieros 2016 & $\begin{array}{c}\text { Total } \\
\text { Segmento } \\
5\end{array}$ & $\begin{array}{l}\text { Total } \\
\text { SFPS }\end{array}$ \\
\hline ACTIVOS & 111,97 & $8.440,32$ & ACTIVOS & 127,16 & $9.514,82$ \\
\hline PASIVOS & 85,97 & $7.090,41$ & PASIVOS & 95,04 & $8.027,76$ \\
\hline PATRIMONIO & 26,00 & $1.349,92$ & PATRIMONIO & 32,12 & $1.487,06$ \\
\hline CAPITAL SOCIAL & 18,29 & 686,61 & CAPITAL SOCIAL & 19,40 & 692,45 \\
\hline CRÉDITOS (CUENTA 14) & 78,22 & $6.298,90$ & CRÉDITOS (CUENTA 14) & 78,37 & $6.165,43$ \\
\hline DEPÓSITOS (CUENTA 21) & 73,26 & $6.395,47$ & DEPÓSITOS (CUENTA 21) & 81,47 & $7.410,51$ \\
\hline $\begin{array}{l}\text { MOROSIDAD DE LA } \\
\text { CARTERA TOTAL }\end{array}$ & $17,49 \%$ & $8,23 \%$ & $\begin{array}{l}\text { MOROSIDAD DE LA } \\
\text { CARTERA TOTAL }\end{array}$ & $16,16 \%$ & $7,40 \%$ \\
\hline LIQUIDEZ & & & LIQUIDEZ & & \\
\hline $\begin{array}{l}\text { FONDOS DISPONIBLES / } \\
\text { TOTAL DEPÓSITOS A } \\
\text { CORTO PLAZO }\end{array}$ & $32,5 \%$ & $38,0 \%$ & $\begin{array}{l}\text { FONDOS DISPONIBLES / } \\
\text { TOTAL DEPÓSITOS A } \\
\text { CORTO PLAZO }\end{array}$ & $50,6 \%$ & $28,8 \%$ \\
\hline $\begin{array}{l}\text { VULNERABILIDAD DEL } \\
\text { PATRIMONIO }\end{array}$ & & & $\begin{array}{l}\text { VULNERABILIDAD DEL } \\
\text { PATRIMONIO }\end{array}$ & & \\
\hline $\begin{array}{l}\text { CARTERA IMPRODUCTIVA } \\
\text { / PATRIMONIO }\end{array}$ & $54,9 \%$ & $40,7 \%$ & $\begin{array}{l}\text { CARTERA IMPRODUCTIVA } \\
\text { / PATRIMONIO }\end{array}$ & $41,7 \%$ & $32,9 \%$ \\
\hline
\end{tabular}

Tabla 9.- Estados Financieros Comparativos

Se toma en cuenta también que el análisis de indicadores para el periodo 2015 tuvo un total de 802 cooperativas y para el 2016, 696 cooperativas, disminuyendo el 13,21\%. 


\section{2.- Entidades del SFEPS en liquidación}

Desde el año 2013 y según el catastro hasta diciembre de 2016, se encuentran en procesos de liquidación 192 cooperativas de ahorro y crédito, dato confirmado por la Corporación de Seguro de Depósitos (Cosede). Cifra alarmante ya que genera desconfianza en el Sistema Financiero Popular y Solidario, más aún si no se conocen oportunamente los motivos de estas liquidaciones. [3]

\section{Características del proceso de liquidación en el SFPS}

*Incumplimientos Regulatorios

*Créditos vinculados

* Licitud de Fondos

*Requerimiento de Patrimonio

*Salud Financiera precaria

*Fusiones

*Entidades con Activos menores a USD 1000.000,00 (un millón de dólares)

Tabla 10.- Cooperativas de Ahorro y Crédito en Liquidación x segmento

\begin{tabular}{lrr}
\hline \multicolumn{3}{c}{ COACS en Liquidación } \\
\hline Segmento 1 & 1 & $0,52 \%$ \\
Segmento 2 & 1 & $0,52 \%$ \\
Segmento 3 & 5 & $2,60 \%$ \\
Segmento 4 & 17 & $8,85 \%$ \\
Segmento 5 & 168 & $87,50 \%$ \\
Total & 192 & $100 \%$ \\
\hline
\end{tabular}

Fuente: Catastro SEPS- Autor

En la tabla 8 podemos observar que el segmento 5 tiene el mayor número de COACs en liquidación, contabilizando un número de 168 entidades que representa el 87,50\% del total de cooperativas con este percance. Basta conocer las características que ha tenido este proceso para entender que el segmento 5 es quien genera el riesgo para el SFPS.

Este proceso en cifras reales se pudo conocer a través del pago de seguros por parte de la COSEDE donde se indicó que se desembolsó USD 29000. 000,00 para un número de 274995 depositantes, desde el año 2013 hasta el cierre del 31 de diciembre de 2016, valor que se incrementaría, de acuerdo a los funcionarios de la Corporación, teniendo en cuenta que el máximo monto de cobertura es de USD $11.000,00$

Para el cierre del mes de agosto de 2017 se cuentan con cuatro entidades más en el mismo proceso [4]

\section{1.- Calificación de Riesgos}

Se tiene dentro de la base de datos de la Dirección Nacional de Información, Técnica y Estadísticas, una calificación de riesgos que incluyen únicamente a 44 cooperativas del SFPS que representa el $6,43 \%$ del total de entidades, que se detalla a continuación: 
Tabla 11.- Calificación de Riesgos

\begin{tabular}{lrrr}
\hline Calificación de Riesgo & 2017 & 2016 & 2015 \\
\hline AAA & 0 & 0 & 0 \\
AA & 6 & 7 & 7 \\
A & 17 & 19 & 17 \\
BBB & 6 & 4 & 5 \\
BB & 6 & 7 & 7 \\
B & 8 & 6 & 8 \\
C & 1 & 1 & 0 \\
Número de Entidades & 44 & 44 & 44 \\
\hline
\end{tabular}

Fuente: Dirección Nacional de Información Técnica y Estadísticas - Autor

Esta calificación representa a los tres primeros segmentos y para las entidades del segmento 4 y 5 las calificaciones son inexistentes. Así mismo se puede analizar que ninguna entidad pasa a una calificación AAA, ya que las instituciones tienen algunas deficiencias significativas. Su capacidad para manejar un mayor deterioro está por debajo de las instituciones con mejor calificación.

\section{Resultados}

El SFPS representa para el país la economía inclusiva, que se distingue por sus principios de solidaridad e integración de las comunidades. Cuando se da la segmentación para el año 2015 se logra identificar entidades que no se encontraban reguladas por el sistema financiero, ya que para el 2014 sólo se contaban con 4 segmentos identificados por su ubicación y número de socios. La segmentación del 2015 da como resultado un estudio avanzado sobre la cantidad y número de activos, sin embargo, su aplicación extingue a varias organizaciones sin embargo las fusiones de las entidades se venían realizando desde el año 2013, por disposiciones de administrativos y voluntad de los socios, medida que no indica un buen estado financiero, pero se superpone a la posibilidad de la extinción de las mismas.

Los activos del SFPS han crecido en un 12,03\% en relación al periodo 2015 , los pasivos se incrementan en un 13,22\%, el capital social crece en un $0,87 \%$ y el patrimonio se incrementa en un 10,16\%. No obstante, para el periodo 2015 se contaba con un 13,21\% más en número de organizaciones, lo que en cifras representaría la existencia de 106 entidades más.

En el segmento 5 se cuenta con los siguientes porcentajes de crecimiento que guarda relación al total del periodo 2016: -Activos: 13,56\% Pasivos: 10,55\% Patrimonio: 23,52\% Capital Social: $6,08 \%$.

-Cartera colocada: para el año 2016 se incrementa el valor de la cartera neta en un 0,03\%. Los tipos de crédito que otorga este segmento en mayor porcentaje son: El Microcrédito con 50,92\% y el de Consumo prioritario con el 23,86\%

-Morosidad de la Cartera: para el periodo 2016 es del 16,16\% decreciendo un 1,33\% del anterior periodo. Sin embargo, sobrepasa el promedio para el sistema que es de 7,40\%. La morosidad se da en los siguientes tipos de crédito: Crédito Inmobiliario 29,12\%, Crédito Comercial Prioritario con 24,79\%, Microcrédito 18,10\%, Crédito Comercial Ordinario 10,20\%. Con este indicador financiero identificamos el problema del segmento 5, ya que, si bien la cartera se ha colocado en un mayor porcentaje frente al periodo anterior, la morosidad crece superando un promedio establecido, y su concesión para microcréditos y consumo prioritario se ven afectados por una elevada morosidad 
que repercute en la pérdida de capital para la empresa, desencadenando liquidaciones por no poder cumplir con un patrimonio establecido en la ley o no sujetarse a la entrega oportuna de sus balances al ente de control. Entre los periodos 2015 y 2016 se cuentan con 168 entidades en liquidación disminuyendo el $13,21 \%$ de entidades dentro del SFPS.

Se puede entender problemas puntuales dentro de la morosidad, como un estudio precario en la autorización del crédito, sistemas de Control inadecuados; créditos Vinculados; seguimiento en el proceso de crédito no favorable. Por otra parte, la calificación de riesgos emitidas por las diferentes empresas calificadoras está dadas sólo para algunas de las entidades de los segmentos 1,2,3 es decir el 30,6\% del total de los segmentos. Esta calificación está dada en función al crédito concedido y la salud financiera de las instituciones; sin embargo, la gestión del riesgo en una entidad actualmente abarca: el riesgo operacional, el riesgo legal, reputacional y de contagio. Al establecer un número importante de entidades cerradas o en procesos de liquidación nos enfrentamos a un riesgo reputacional para el SFPS ya que la masa o cartera de socios ve comprometidos sus recursos en caso de que las instituciones tengan que cesar sus funciones, por esta razón es importante que la SEPS establezca mecanismos eficientes que contrarresten estos riesgos y no decrezca el número de depositantes dentro del SFPS.

La existencia del segmento 5 está justificado dentro del SFPS, ya que consolida, en menor número, la importancia de la asociación comunitaria y pequeña del sector económico del país. No obstante, es necesario tener una hoja de ruta clara para el fortalecimiento del sector en base a 4 objetivos: el fortalecimiento patrimonial de las cooperativas, el incremento de la gestión integral de riesgo, la gestión de riesgo de crédito y los cumplimientos de solvencia.

\section{Conclusiones}

- El financiamiento es una poderosa herramienta para dinamizar la economía popular y solidaria, ya que promueve el equilibrio de los ingresos y constituye un mecanismo que permite el flujo de fondos a los sectores más relegados del país para promover su desarrollo. Las cooperativas de ahorro y crédito realizan actividades de intermediación financiera, basadas fundamentalmente en la fortaleza del ahorro de los depositantes o socios, por lo que, a diferencia del sector financiero formal, pueden actuar como impulsores de emprendimientos dinámicos y de mayor potencial, mediante el financiamiento de proyectos para el desarrollo de las pequeñas empresas

- La segmentación de las cooperativas de Ahorro y crédito es un gran logro dentro del SFPS ya que posibilita a los entes de control aplicar políticas y reglas claras para cada segmento, según las necesidades y en relación a los activos que se manejan en las entidades. El segmento 5 del SFPS se constituye con cooperativas de ahorro y crédito, cajas y bancos comunales, el mismo que desarrolla y presta sus servicios de intermediación financiera para las MiPymes del país, concentradas en su mayoría en la región Sierra, donde las actividades económicas principales son primarias. 


\section{Referencias bibliográficas}

[1]. SEPS. Superintendencia de Economía Popular y Solidaria. [En línea] diciembre de 2016. [Citado el: 28 de agosto de 2017.] http://www.seps.gob.ec/.

[2]. Actualícese. Actualícese. Actualícese. [En línea] agosto de 2015. [Citado el: 28 de agosto de 2017.] http://actualicese.com/2015/02/26/definicion-de-indicadores-financieros/.

[3]. SEPS. Superintendencia de Economía Popular y Solidaria. Listado de Liquidaciones. [En línea] 20 de agosto de 2017. [Citado el: 28 de agosto de 2017.] www.seps.gob.ec.

[4]. —. Boletín Financiero al 31 de diciembre de 2016. Quito: s.n., 2016. 
PARA CITAR EL ARTÍCULO INDEXADO.

Rodríguez Solarte, Ángel, Chávez Rojas, J., Ávalos Peñafiel, V., \& Sánchez Chávez, R. (2019). Sector Financiero Popular y Solidario: cooperativas de ahorro y crédito del segmento 5 en Ecuador. Ciencia Digital, 3(3.2.1), 217-227. https://doi.org/10.33262/cienciadigital.v3i3.3.809

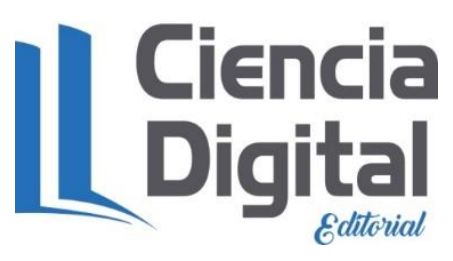

El artículo que se publica es de exclusiva responsabilidad de los autores y no necesariamente reflejan el pensamiento de la Revista Ciencia Digital.

El artículo queda en propiedad de la revista y, por tanto, su publicación parcial y/o total en otro medio tiene que ser autorizado por el director de la Revista Ciencia Digital.
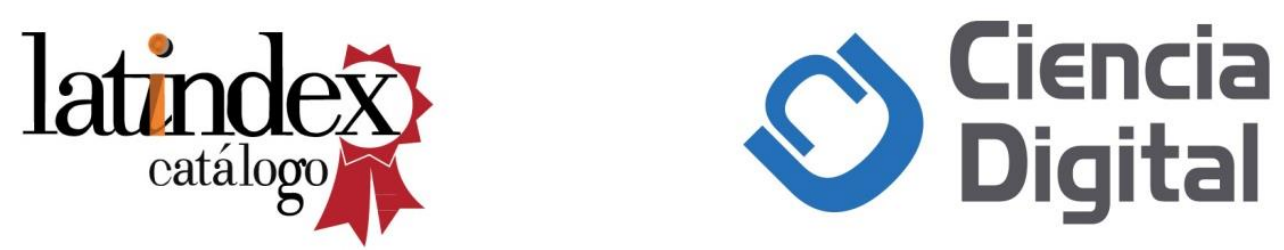